\title{
Concordancia de la utilización de teriparatide o ácido zoledrónico en pacientes con osteoporosis con recomendaciones internacionales, en una empresa de salud de Colombia
}

\author{
Concordance in the use of teriparatide or zoledronic \\ acid in patients with osteoporosis with international \\ recommendations, in a health company of Colombia
}

Yuly Henao-Z $Z^{1,2}$; Piedad-L Botero-S ${ }^{1}$; Ilsa-Y Parrado-F',3; Pedro Amariles ${ }^{2}$

\begin{abstract}
Forma de citar: Henao Y, Botero P, Parrado I, Amariles P. Concordancia de la utilización de teriparatide o ácido zoledrónico en pacientes con osteoporosis con recomendaciones internacionales, en una empresa de salud de Colombia. Rev Univ Ind Santander Salud. 2017; 49(3): 458-468. doi: http://dx.doi.org/10.18273/revsal.v49n3-2017004 @) (1)
\end{abstract}

\section{Resumen}

Introducción: Los bifosfonatos son la primera línea de tratamiento para la osteoporosis; siendo el zolendronato el más efectivo. La teriparatide (agente anabólico), dado su alto costo, debería restringirse al tratamiento de pacientes más afectados y luego de un adecuado tratamiento con bifosfonatos. Objetivo: Establecer el porcentaje de pacientes con osteoporosis, en tratamiento con teriparatide o ácido zoledrónico en Colombia, que se ajusta a recomendaciones de organizaciones internacionales. Método: Estudio de utilización de medicamentos retrospectivo, enero 2012-diciembre 2015, en pacientes con osteoporosis y en tratamiento con teriparatide o ácido zoledrónico. Se revisó información sociodemográfica y clínica, y el grado de concordancia del uso de teriparatide o ácido zoledrónico con recomendaciones de tres guías clínicas internacionales: National Osteoporosis Foundation, National Institute for Health and Clinical Excellence y Scottish Intercollegiate Guidelines Network. Resultados: Se incluyeron 103 pacientes, edad promedio en años (desviación estándar) 78 (10,4), 74,8\% mujeres. La osteoporosis posmenopáusica $(61,2 \%)$ y osteoporosis no especificada $(20,4 \%)$, ambas con o sin fractura patológica, fueron los diagnósticos principales. En el 36,9\%, la utilización de teriparatide y ácido zoledrónico fue acorde a las recomendaciones de las guías revisadas. La utilización de estos medicamentos en diagnósticos diferentes a osteoporosis posmenopáusica $(43,7 \%)$ o como farmacoterapia inicial $(36,9 \%)$ fueron las principales causas para ausencia de concordancia. Conclusiones: Sólo en el 36,9\% de los casos revisados, la utilización de teriparatide o ácido zoledrónico se

1. Universidad Tecnológica de Pereira. Pereira, Colombia.

2. Universidad de Antioquia. Medellín, Colombia.

3. Universitat Pompeu Fabra. Barcelona, España.

Correspondencia: Yuly Henao Zapata. Dirección: Calle 30 A No 6-22 Edificio San Martin, Oficina 902. Bogotá- Colombia.

Correo electrónico: yulyh@audifarma.com.co. Teléfono: (+74) 3193428847. 
ajusta a las guías de organizaciones internacionales. La ausencia de concordancia se debe, especialmente a uso en diagnósticos diferentes a osteoporosis posmenopáusica y como farmacoterapia inicial.

Palabras clave: Osteoporosis; Teriparatide; Conservadores de la densidad ósea; Utilización de medicamentos; Farmacoepidemiología.

\begin{abstract}
Introduction: Bisphosphonates are the first-line treatment of osteoporosis in which zoledronate is the most effective. Teriparatide (anabolic agent), due to high cost, should be restricted to the treatment of the most affected patients and after adequate treatment with bisphosphonates. Objective: To establish the percentage of patients with osteoporosis, in treatment with teriparatide or zoledronic acid in Colombia, that meet international organizations recommendations. Method: Retrospective drug utilization study, January 2012-December 2015, in patients with osteoporosis and treatment with teriparatide or zoledronic acid. The socio-demographic and clinical information and the degree of concordance of teriparatide or zoledronic acid use with recommendations from three international clinical guidelines were reviewed: National Osteoporosis Foundation, National Institute for Health and Clinical Excellence, and Scottish Intercollegiate Guidelines Network. Results: 103 patients were included, average age in years (standard deviation) 78 (10.4), 74.8\% women. Postmenopausal osteoporosis $(61.2 \%)$ and unspecified osteoporosis $(20.4 \%)$, both with or without pathological fracture, were the main diagnoses. In $36.9 \%$, the use of teriparatide and zoledronic acid was accord with the guidelines recommendations revised. The use of these drugs in diagnoses different to postmenopausal osteoporosis $(43.7 \%)$ or initial pharmacotherapy $(36.9 \%)$ were the main causes for concordance absence. Conclusions: Only $36.9 \%$ of the reviewed cases, the use of teriparatide or zoledronic acid was accord with international organizations guideliness. Concordance absence is due especially both to the use in diagnoses different to postmenopausal osteoporosis and initial pharmacotherapy.
\end{abstract}

Keywords: Osteoporosis; Teriparatide; Bone density conservation agents; Drug utilization; Pharmacoepidemiology.

\section{Introducción}

La osteoporosis es un problema se salud creciente en la población adulto mayor. Epidemiológicamente, los datos globales evidencian, de forma consistente, un aumento con la edad en la incidencia anual de fracturas $^{1}$, debido a que la masa y densidad ósea tiende a caer, especialmente en las mujeres posmenopáusicas. Esta situación puede progresar y, con ello, aumentar significativamente el riesgo de fractura, lo que tiene efectos notorios en la discapacidad, la reducción de la calidad de vida y la mortalidad ${ }^{2}$.

En Colombia, para el año 2012 se estimó que 1.423.559 mujeres de 50 años vivían con osteoporosis, cifras proyectadas a los 1.573 .173 casos, en el 2020, y los 2.101.000 casos en el año $2050^{3}$. En este sentido, un estudio realizado por el Instituto Nacional de Salud de Colombia, mostró un incremento notorio de la osteoporosis en las mujeres colombianas durante la quinta y sexta década de vida, además de una prevalencia más elevada, en comparación con datos internacionales para el mismo grupo etario ${ }^{4}$. Por su parte, en mujeres colombianas de 50 años o más, se estima que anualmente ocurren unas 8.000 a 10.000 fracturas de cadera (cifra que para el 2020 alcanzaría las 11.500),
284.711 fracturas vertebrales y 854.135 fracturas osteoporóticas en otros sitios $^{5}$. No obstante, dichas estimaciones se basan en los datos de las compañías de seguro de salud, proyectadas a las estadísticas generales de la población. Por tanto, en Colombia el impacto real de la osteoporosis, sobre el estado de salud, la calidad de vida de los pacientes y la carga económica, aún está por establecer.

El uso de medicamentos hace parte de las estrategias terapéuticas para el manejo de la osteoporosis, de ellos, los bifosfonatos son el grupo farmacoterapéutico de primera línea para su prevención y tratamiento ${ }^{1}$. En estudios clínicos controlados se ha evidenciado una reducción significativa en la incidencia de fracturas vertebrales y no vertebrales para el alendronato, risedronato, ibandronato y zolendronato (o ácido zoledrónico $)^{6-11}$ comparado con placebo.

El zolendronato (Zol), en comparación con placebo, muestra la mayor tasa de reducción de las fracturas vertebrales en pacientes con osteoporosis ${ }^{1}$. Adicionalmente, su vía de administración intravenosa (con la cual se reduce efectos adversos gastrointestinales propios de este grupo farmacoterapéutico) y su frecuencia anual de administración, favorece la 
adherencia y persistencia con el tratamiento, lo que se asocia con mejores resultados en pacientes con osteoporosis. Por su parte, la teriparatide (TTP), es un medicamento desarrollado mediante tecnología del ADN recombinante, es idéntica a la secuencia N-terminal de 34 aminoácidos de la hormona paratiroidea humana endógena. Fue aprobado en el año 2002 por la FDA (Food and Drug Administration, por sus siglas en inglés), siendo el primer fármaco de una nueva clase de medicamentos para la osteoporosis, conocidos como agentes anabólicos ${ }^{12}$. Teriparatide es un agente potente que estimula directamente la formación de nuevo tejido óseo en pacientes con osteoporosis severa $^{13}$, reduce la incidencia de fracturas esqueléticas a niveles equivalentes, o superiores a los bifosfonatos. Sin embargo, dado el alto costo de esta medicación, su uso debería restringirse al tratamiento de pacientes más graves (más de una fractura por fragilidad y densidad mineral ósea muy baja, T-score $<-3,5$ ) o en pacientes que sufren nuevas fracturas, luego de dos o más años de un adecuado tratamiento con un bifosfonato ${ }^{14}$.

En el contexto de Colombia, la información disponible sobre el patrón de prescripción de estos medicamentos, es limitada. En este sentido, en la bibliografía consultada, sólo se identificó un estudio, realizado en una institución de salud de Bogotá, el cual se orientó al manejo farmacológico general de la osteoporosis y osteopenia ${ }^{15}$, y no fue específico para TTP y Zol. Por tanto, el objetivo de este trabajo fue establecer el porcentaje de pacientes con osteoporosis, en tratamiento con teriparatide o ácido zoledrónico en una empresa de salud de Colombia, entre enero de 2012 y diciembre de 2015, que se ajusta a las recomendaciones de organizaciones internacionales reconocidas.

\section{Metodología}

Tipo de estudio y población objeto: Se realizó un estudio de utilización de medicamentos de tipo descriptivo retrospectivo, en pacientes con diagnóstico de osteoporosis, pertenecientes a una empresa de salud de Colombia, durante el periodo enero de 2012 a diciembre de 2015.

Muestra: Se utilizó información de una base de datos poblacional de 1.9 millones de personas que corresponden aproximadamente al 9,3\% de la población activa afiliada al régimen contributivo del Sistema General de Seguridad Social en Salud de Colombia. Los datos fueron de 22 ciudades del país, incluyendo las 4 ciudades principales (Bogotá, Medellín, Cali y Barranquilla). Con esta información, se seleccionaron los pacientes con dispensaciones de ácido zoledrónico o teriparatide, durante el periodo de estudio, en total 762 pacientes. Acorde con los datos de consumo reportados para el año 2013 en Colombia para ambos medicamentos (Zol:0,42\%; TTP; 5,86\%) ${ }^{16}$, se realizó un muestreo sistemático con un margen de error del $5 \%$, nivel de confianza del $95 \%$ y con la frecuencia de consumo de TTP (por ser más alto que el del Zol). Por tanto, el tamaño de muestra fue de 77 pacientes; no obstante, se consideró ampliar el tamaño de la muestra a mínimo 100 pacientes, con el fin de contrarrestar posible falta de información en los registros utilizados como fuente de información. Posteriormente, del listado de los 762 pacientes, se seleccionaron, de forma consecutiva, a los pacientes ubicados en las posiciones $7,14,21$, y sucesivos de la base de datos.

\section{Fuentes de información:}

- Registros de dispensaciones (RD): La información de la dispensación de medicamentos es almacenada sobre una plataforma LINUX, en una base de datos POSTGRESQL y utilizando el aplicativo DELPHI para Windows. A partir de esta base de datos se generó la información por cliente institucional, por usuario y por medicamento. Posteriormente, la información fue validada por el equipo de ingenieros de Audifarma S.A. y revisada por los autores, para verificar la consistencia de los datos.

- Historia clínica (HC): Se accedió a los registros de $\mathrm{HC}$ de la empresa de salud, incluyendo consulta externa por medicina general, consulta médica prioritaria, consulta por medicina especializada (algunas sedes) y registros de paraclínicos.

\section{Variables:}

Se construyó una base de datos en Microsoft Excel (Windows 7) para recopilar la información relevante de los RD y las variables clínicas y sociodemográficas consignadas en la HC. De este modo, las variables independientes fueron:

- Socio-demográficas y clínicas: Edad, sexo, ciudad y diagnóstico principal, según la Clasificación Internacional de Enfermedades versión 10 (CIE10). Fuente: HC.

- Uso de medicamentos para la osteoporosis: En la base de datos de RD se identificó el medicamento utilizado por cada paciente (TTP o Zol), la dosis, frecuencia y tiempo de tratamiento en meses. Adicionalmente, para evaluar si el paciente tuvo o no tratamiento previo con otros medicamentos antiosteoporóticos, se realizó una búsqueda, en el $\mathrm{RD}$, un año retrospectivo, a partir de la fecha 
de inicio de alguno de los dos medicamentos de interés, y se complementó con la información consignada en la HC.

Con el propósito de realizar cálculo del riesgo de fractura a 10 años, se identificaron los factores de riesgo propuestos por la Organización Mundial de la Salud (WHO Fracture Risk Assessment Tool- FRAX $\left.{ }^{\circledR}\right)^{17}$. Por ello, también se incluyeron las siguientes variables:

- Comorbilidades:Artritis Reumatoide-Osteoporosis secundaria (SI/NO). Fuente: HC.

- Fractura osteoporótica previa (incluyendo fractura vertebral morfométrica) (SI/NO). Fuente HC.

- Historia de los padres de fractura de cadera: (SI/ NO). Fuente HC.

- Densitometría mineral ósea (DMO) cuello femoral y lumbar, previo al inicio del tratamiento con TTP o Zol: se utilizó T-score basado en los valores de referencia NHANES III para mujeres ${ }^{18}$. Fuente HC.

- Tabaquismo actual: (SI/NO) Fuente: HC.

- Peso: kilogramos. Fuente HC.

- Estatura: centímetros. Fuente HC.

- Consumo de alcohol (tres o más bebidas/día): Una dosis corresponde a cerveza $(285 \mathrm{ml})$, una copa de licor (30ml), una copa de vino de tamaño mediano $(120 \mathrm{ml})$, ó 1 copa de aperitivo $(60 \mathrm{ml})$. Fuente HC.

- Uso de glucocorticoides orales: dosis superiores a los $5 \mathrm{mg} /$ día de prednisona o su equivalente durante tres o más meses. Fuente: RD.

Finalmente, se evaluaron los patrones o hábitos de prescripción, mediante la comparación con los parámetros establecidos por las siguientes Guías de manejo clínico (variables dependientes): National Osteoporosis Foundation (NOF) ${ }^{19}$, National Institute for Health and Clinical Excellence (NICE) ${ }^{20} y$ Scottish Intercollegiate Guidelines Network $(S I G N)^{21}$. La información clave relacionada con dichas guías se detalla en la Tabla 1, el no cumplimiento de algún parámetro se consideró como ausencia de concordancia, y se registró en la base de datos como una variable categórica dicotómica (cumple:1; No cumple: 0 ).

En este sentido, en el caso de la guía NICE por ejemplo, la cual sólo recomienda el uso de TTP para mujeres con diagnóstico de osteoporosis posmenopáusica, se consideraron como no concordantes las prescripciones de TTP en pacientes de sexo masculino o en mujeres con otro diagnóstico.

Tabla 1. Recomendaciones de las guías internacionales para el manejo de la osteoporosis

\begin{tabular}{|c|c|}
\hline Guía & Teriparatide \\
\hline & $\begin{array}{l}\text { Indicaciones generales } \mathbf{N O F}^{\text {a }} \text { : Mujeres postmenopáusicas y hombres }>\text { de } 50 \text { años, con alguno de los siguientes aspectos: } \\
\text {-Fractura de cadera o vertebral (clínica o morfométrica). } \\
\text {-T-score } \leq-2,5 \text { en cuello o columna lumbar asociado a OP. } \\
\text { - Baja masa ósea (T-score entre -1 y }-2,5 \text { en cuello femoral o columna) y probabilidad a } 10 \text { años de fractura de cadera } \geq 3 \% \text { o } \\
\text { una probabilidad a } 10 \text { años de cualquier fractura relacionada con OP } \geq 20 \% \text { basado en el algoritmo de la OMS. }\end{array}$ \\
\hline NOF $^{\mathrm{a} 19}$ & $\begin{array}{ll}\text {-Tratamiento de OP posmenopáusica y hombres con alto riesgo de } & \text {-Tratamiento y prevención de OP posmenopáusica. } \\
\text { fractura. } & \text {-Tratamiento y prevención OP por uso de } \\
\text {-Tratamiento OP por uso de glucocorticoides con alto riesgo de fractura. } & \text { glucocorticoides } \geq 12 \text { meses. } \\
\text { Hombres con osteoporosis primaria o hipogonadal en alto riesgo de } & \text {-Prevención de fracturas nuevas en pacientes con } \\
\text { fracturar } & \text { un trauma reciente leve de fractura de cadera. }\end{array}$ \\
\hline NICE $^{\mathrm{b} 20}$ & $\begin{array}{l}\text { Opción alternativa para prevención secundaria de fracturas } \\
\text { por fragilidad osteoporóticas en mujeres posmenopáusicas: } \\
\text {-Incapaces de tomar o contraindicación de alendronato y } \\
\text { risedronato o etidronatoranelato de estroncio (náuseas o diarrea } \\
\text { persistente, que requiera la interrupción del tratamiento), o no No especifica } \\
\text { han tenido respuesta satisfactoria con estos medicamentos. } \\
-\mathrm{Y} \geq 65 \text { años y T-score } \leq-4,0 \mathrm{SD} \text { o T-score } \leq-3,5 \mathrm{SD} \text {, además }>2 \text { fracturas; } \\
\text { o entre } 55-64 \text { años y t T-score } \leq-4 \mathrm{SD} \text { y }>2 \text { fracturas. }\end{array}$ \\
\hline SIGN $^{\mathrm{c} 21}$ & $\begin{array}{l}\text { Segunda línea en OP postmenopáusica, después de una -Mujeres posmenopáusicas con alto riesgo } \\
\text { evaluación del riesgo de fractura incluyendo la medición de de fracturas, cuando no es adecuado o } \\
\text { la DMO. Se ha demostrado eficacia en la reducción vertebral no toleran opciones de tratamiento oral } \\
\text { y fracturas no vertebrales en mujeres posmenopáusicas con -No recomendado en hombres con riesgo } \\
\text { fracturas vertebrales anteriores; en particular, en OP severa. incrementado de fractura, incluyendo aquellos con } \\
\text {-No recomendado en hombres, ni en OP por glucocorticoides sistémicos una fractura de cadera reciente de bajo impacto. } \\
\text { tanto en hombre como mujeres. }\end{array}$ \\
\hline
\end{tabular}

${ }^{a}$ National Osteoporosis Foundation

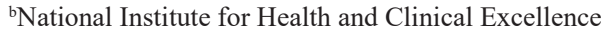

${ }^{\mathrm{c} S}$ cottish Intercollegiate Guidelines Network

*Siglas: OP (Osteoporosis); DMO (Densitometría mineral ósea); SD (desviación estándar). 


\section{Análisis estadístico}

El registro de la información se realizó en una base de datos elaborada en Microsoft Excel ${ }^{\circledR}$ (Windows 7), la cual fue validada y revisada por los autores para minimizar sesgos de digitación, selección e información. Por su parte, el análisis estadístico se realizó con el programa informático IBM SPSS versión 24 para Windows (SPSS Inc., Chicago, Illinois). Acorde con el objetivo del trabajo, se calcularon la estadística descriptiva, las frecuencias, promedios, rangos, intervalos de confianza (variables cuantitativas) y desviación estándar (DE) de las variables evaluadas. Adicionalmente, se realizaron comparaciones de las variables asociadas a riesgo de fractura, definidas por la $\mathrm{OMS}^{17}$, al porcentaje de ausencia de concordancia, estratificado por sexo, y a las variables ordinales: DMO cuello femoral o de cadera y riesgo de fractura mayor o de cadera, acorde con el medicamento: TTP O ZOL. Para ello, se utilizaron la prueba de Chi-cuadrado para proporciones, o T-student para medias - muestras independientes, considerando un valor de $\mathrm{p}<0,05$ como estadísticamente significativo.

\section{Resultados}

Durante el periodo enero de 2012 a diciembre de 2015, se identificaron 762 pacientes en tratamiento con Zol ( $\mathrm{n}=518)$ o TTP $(\mathrm{n}=256)$. De este grupo, a 103 pacientes (TTP:51; Zol:52), seleccionados de forma aleatoria, se les revisó la historia clínica y el historial de dispensaciones. Este valor de 103 fue superior a los 77 (definidos por el muestreo sistemático) y a los 100 (establecidos para contrarrestar la posible falta de información en las fuentes de información).

Las características sociodemográficas y el diagnóstico principal, según CIE10 por medicamento se resumen en la Tabla 2. Acorde con los diagnósticos consignados por $\mathrm{HC}$, el $57,3 \%$ de la población no presentó fracturas previo al inicio del tratamiento.

Tabla 2. Características sociodemográficas y diagnóstico principal.

\begin{tabular}{|c|c|c|c|}
\hline \multicolumn{2}{|r|}{ Variable } & Teriparatide $(n=51)$ & Zoledrónico $(\mathrm{n}=52)$ \\
\hline \multirow{3}{*}{ Edad (años) } & Promedio (DE) & $76(12,3)$ & $73(8,0)$ \\
\hline & $\mathrm{IC}$ & 72,5 a 79,2 & 71,1 a 75,4 \\
\hline & Rango & 21 a 94 & 56 a 90 \\
\hline \multirow{2}{*}{ Sexo } & Femenino, $\mathrm{n}(\%)$ & $45(88,2)$ & $32(61,5)$ \\
\hline & Masculino, n (\%) & $6(11,8)$ & $20(38,5)$ \\
\hline \multirow{3}{*}{ IMC (Kg/m2) } & Promedio (DE) & $23,4(3,5)$ & $24,3(4,0)$ \\
\hline & $\mathrm{IC}$ & 22,5 a 24,3 & 23,1 a 25,4 \\
\hline & Rango & 14,6 a 31,4 & 15,6 a 35,6 \\
\hline \multirow{6}{*}{$\begin{array}{l}\text { Distribución por } \\
\text { ciudades }\end{array}$} & Bogotá, n (\%) & $29(56,9)$ & $40(76,9)$ \\
\hline & Cali, n (\%) & $0(0,0)$ & $2(3,8)$ \\
\hline & Medellín, n (\%) & $11(21,6)$ & $1(1,9)$ \\
\hline & Manizales, n (\%) & $3(5,9)$ & $6(11,5)$ \\
\hline & Villavicencio, n (\%) & $3(5,9)$ & $1(1,9)$ \\
\hline & Otras, n $(\%)$ & $5(9.8)$ & $2(3,8)$ \\
\hline \multirow{14}{*}{ Diagnóstico (CIE10) - } & OP en enfermedades clasificadas en otra parte, n $(\%)$ & $0(0,0)$ & $1(1,9)$ \\
\hline & OP en trastornos endocrinos, $\mathrm{n}(\%)$ & $0(0,0)$ & $1(1,9)$ \\
\hline & OP idiopática, con fractura patológica, n (\%) & $0(0,0)$ & $4(7,7)$ \\
\hline & OP idiopática, sin fractura patológica, n (\%) & $0(0,00$ & $4(7,7)$ \\
\hline & OP inducida por drogas, con fractura patológica, n (\%) & $0(0,0)$ & $1(1,9)$ \\
\hline & OP inducida por drogas, sin fractura patológica, $\mathrm{n}(\%)$ & $1(2,0)$ & $1(1,9)$ \\
\hline & OP localizada [lequesne], sin fractura patológica, n (\%) & $0(0,0)$ & $1(1,9)$ \\
\hline & OP no especificada, con fractura patológica, n (\%) & $8(15,7)$ & $1(1,9)$ \\
\hline & OP no especificada, sin fractura patológica, n (\%) & $2(3,9)$ & $10(19,2)$ \\
\hline & OP postmenopáusica, con fractura patológica, n (\%) & $32(62,7)$ & $5(9,6)$ \\
\hline & OP postmenopáusica, sin fractura patológica, n (\%) & $6(11,8)$ & $20(38,5)$ \\
\hline & OP postooforectomia, con fractura patológica, $\mathrm{n}(\%)$ & $1(2,0)$ & $0(0,0)$ \\
\hline & Otras OP con fractura patológica, n (\%) & $0(0,0)$ & $2(3,9)$ \\
\hline & Otras OP, sin fractura patológica, n (\%) & $1(2,0)$ & $1(1,9)$ \\
\hline
\end{tabular}

Siglas: DE: Desviación estándar, IC: Intervalo de confianza, IMC: Índice de masa corporal, CIE10: Clasificación Internacional de Enfermedades, OP: osteoporosis 
De los 103 pacientes, el 74,8\% fueron mujeres, con una edad promedio de 75 años (IC: 72,7 a 76,6; rango: 21 a 94 años; DE: 10,4); residentes principalmente en las ciudades de Bogotá (67,0\%), Medellín $(11,7 \%)$, Manizales $(8,7 \%)$ y Villavicencio $(3,9 \%)$. Adicionalmente, el 66,0\% fueron mayores de 75 años.

El tiempo de tratamiento promedio para la TTP fue de 10,8 meses (IC: 8,9 a 12,7; rango: 1 a 28 meses; DE: 7,1); y para el Zol de 24,7 meses (IC: 21,4 a 27,9; rango: 24 a 60 meses; DE: 12,2). El 5,9\% de los pacientes que utilizaron TTP, superaban los dos años de tiempo máximo recomendado ${ }^{20}$. En el caso del Zol, el $7,7 \%$ lo hicieron por un periodo superior a tres años. Por su parte, la dosis y frecuencia de administración para ambos medicamentos, en el 100,0\% de los casos se ajustó a lo recomendado ${ }^{19-21}$.

En el $45,1 \%$ y $48,1 \%$ de los pacientes a los que se les inició TTP y Zol, respectivamente, no se documentó, en $\mathrm{RD}$ o $\mathrm{HC}$, tratamiento previo con otro medicamento antiosteoporótico. En el caso particular de TTP, medicamento considerado como de segunda línea, se destaca que el $86,9 \%$ fueron mujeres con osteoporosis posmenopáusica, el $78,3 \%$ mayores de 75 años, el $37,2 \%$ presentaban antecedentes de fracturas previas; y el promedio en la DMO de cadera fue de $-3,2(n=15$; IC:-3,7 a -2,9; rango:-2,1 a $-4,3$; DE:0,7) y DMO lumbar de $-4,1$ (n=13; IC: $-5,1$ a $-3,4$; rango:-2,5 a -7,8; DE:1,4).

Los factores de riesgo evaluados en este estudio se resumen en la Tabla 3, y corresponden a los establecidos por la guía de manejo de la $\mathrm{NOF}^{19}$ y la Organización Mundial de Salud (OMS) para realizar el cálculo del riesgo de fractura a 10 años mediante el algoritmo FRAX ${ }^{\circledR 17}{ }^{17}$, el cual aplica para hombres y mujeres posmenopáusicas mayores de 50 años; dicho cálculo se realizó para cada paciente. Es importante destacar que, la DMO de cuello femoral de 30 pacientes (TTP: 12; Zol: 18), no se reportó en la HC; y adicionalmente, para ningún paciente se registró antecedentes de fracturas de los padres; por ello, este dato se registró como "NO" en el algoritmo FRAX ${ }^{\circledR}$. En la estratificación por sexo de estas variables, sólo se encontró diferencia estadísticamente significativa para la variable "fractura previa", en la cual se observa un porcentaje del $72,6 \%$ de fracturas previas en las mujeres que recibieron TTP comparado con el 15,4\% tratadas con Zol, Tabla 3.

Tabla 3. Factores de riesgo según algoritmo "Fracture Risk Assessment Tool (FRAX®)/Organización Mundial de la Salud (OMS)": estratificado por sexo.

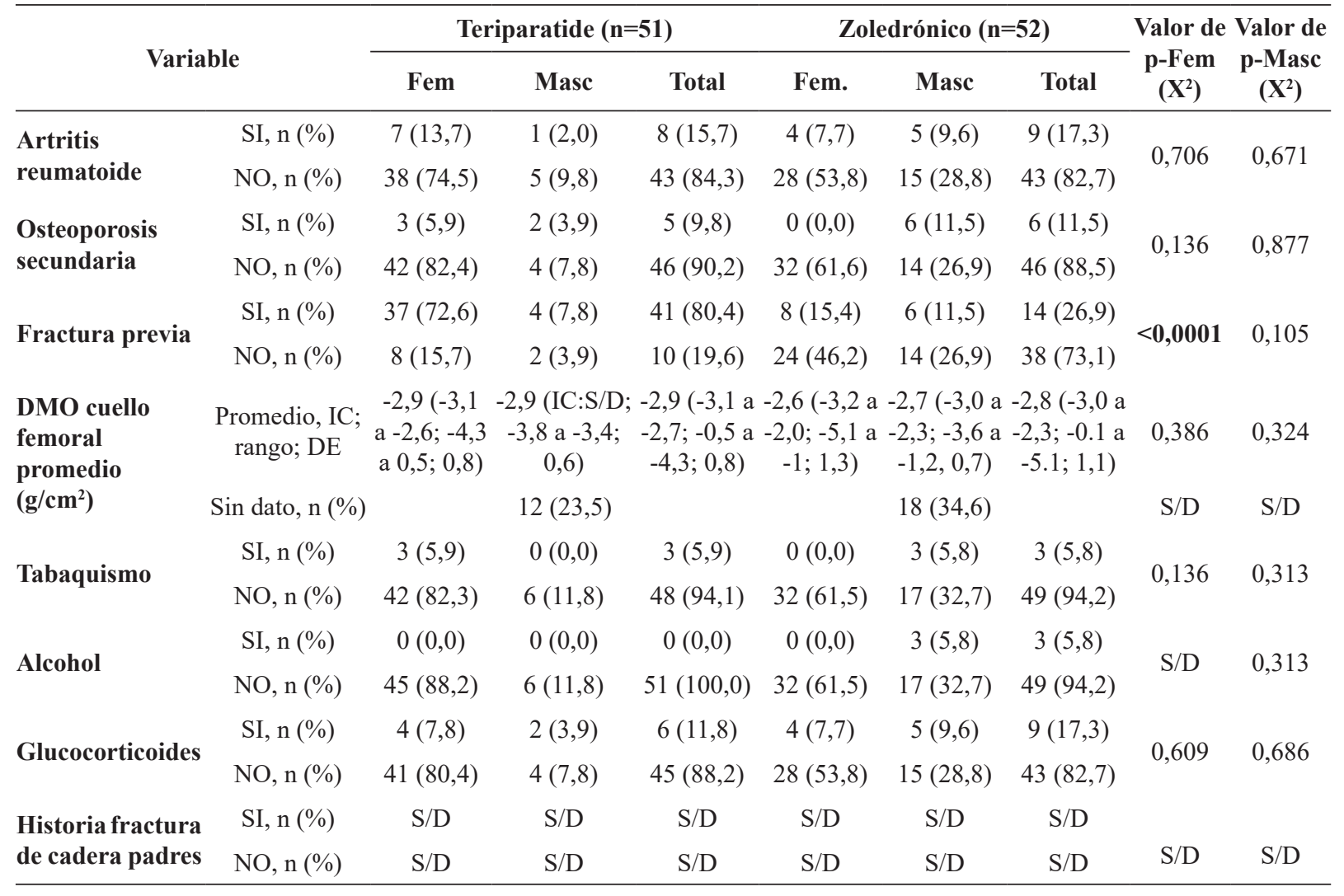

Siglas: Fem: Femenino, Masc: Masculino, S/D: Sin dato. 
El riesgo promedio de osteoporosis o fractura mayor a 10 años fue de $9,2 \%$ (IC: $8,0 \%$ a $10,6 \%$; rango: $3,7 \%$ a $21,0 \%$; DE:4,2) y $7,8 \%$ (IC: $6,0 \%$ a $9,9 \%$; rango: $2,5 \%$ a $22 \%$; DE:5,7) para TTP y Zol, respectivamente; mientras que el de fractura de cadera fue del $4,1 \%$ (IC:3,3\% a 4,9\%; rango: $0,5 \%$ a $11,0 \%$; DE:2,6) y $4,3 \%$ (IC:2,9\% a 5,9\%; rango:0,4\% a 15,0; DE:4,2) para TTP y Zol, respectivamente. No se encontraron diferencias estadísticamente significativas entre ambos medicamentos para el riesgo de fractura mayor (Diferencia media: 1.36, IC: $-1,1$ a 3,$6 ; p=0,264$ ) ni fractura de cadera (Diferencia media: $-0,21$, IC: $-1,9$ a 1,$3 ; p=0,825)$. De igual forma, tampoco se encontraron diferencias por sexo en: riesgo fractura mayor (diferencia media: 1,6, IC: $-0,83$ a 3,99; $\mathrm{p}=0,189$ ), riesgo fractura de cadera (diferencia media: $-0,42$, IC: $-2,3$ a 1,34; $p=0,621)$, Tabla 3.
En el 36,9\% del total de los pacientes, la utilización de teriparatide y ácido zoledrónico fue acorde a las recomendaciones de las guías revisadas ${ }^{19-21}$. En este sentido, las principales causas de no concordancia con dichas guías se describen en la Tabla 4. Para la TTP, acorde con las diferentes guías, las proporciones de concordancia fueron: NOF 96,1\%, NICE $25,5 \%$ y SIGN 62,8\%. Sólo el 37,2\% de los casos no se ajustó a las recomendaciones de la NICE ni SIGN; y en el 3,9\% a ninguna de las tres guías. Por su parte, para el Zol las proporciones de concordancia fueron: NOF 94,2\% y SIGN 48,1\%. Sólo el 5,8\% de los casos no fue indicado el tratamiento para ambas guías (NOF y SIGN). No se encontraron diferencias estadísticamente significativas para la variable ausencia de concordancia por tipo de medicamento (TTP o ZOL) ni por sexo, Tabla 4.

Tabla 4. Causas de ausencia de concordancia del uso de teriparatide y ácido zoledrónico con las recomendaciones de las guías internacionales.

\begin{tabular}{|c|c|c|c|c|c|}
\hline \multirow{3}{*}{ Causa } & \multicolumn{3}{|c|}{ Teriparatide $(\mathrm{n}=51)$} & \multicolumn{2}{|c|}{ Zoledrónico $(\mathrm{n}=52)$} \\
\hline & \multicolumn{3}{|c|}{ Guía } & \multicolumn{2}{|c|}{ Guía* } \\
\hline & NICE $^{\mathrm{b}}$ & NOF $^{\mathrm{a}}$ & SIGN $^{c}$ & NOF $^{a}$ & SIGN $^{\mathrm{c}}$ \\
\hline Osteopenia $(\mathrm{DMO}>-2,5)$ y $\sin$ fractura previa, $\mathrm{n}(\%)$ & N/A & $2(3,9)$ & N/A & $3(5,8)$ & N/A \\
\hline $\mathrm{DMO}>-4, \mathrm{n}(\%)$ & $3(5,9)$ & N/A & N/A & N/A & N/A \\
\hline DMO > -4, No OP-P, n (\%) & $2(3,9)$ & $\mathrm{N} / \mathrm{A}$ & N/A & $\mathrm{N} / \mathrm{A}$ & $\mathrm{N} / \mathrm{A}$ \\
\hline $\mathrm{DMO}>-4, \sin$ fractura previa, $\mathrm{n}(\%)$ & $2(3,9)$ & N/A & $\mathrm{N} / \mathrm{A}$ & N/A & N/A \\
\hline $\mathrm{DMO}>-4$, No OP-P, sin fractura previa, $\mathrm{n}(\%)$ & $1(2,0)$ & N/A & N/A & N/A & N/A \\
\hline $\mathrm{DMO}>-4$, No OP-P, sexo masculino, $\mathrm{n}(\%)$ & $2(3,9)$ & N/A & N/A & N/A & $\mathrm{N} / \mathrm{A}$ \\
\hline $\mathrm{DMO}>-4, \sin$ fractura previa, sin tratamiento previo, $\mathrm{n}(\%)$ & $1(2,0)$ & $\mathrm{N} / \mathrm{A}$ & $\mathrm{N} / \mathrm{A}$ & $\mathrm{N} / \mathrm{A}$ & $\mathrm{N} / \mathrm{A}$ \\
\hline $\mathrm{DMO}>-4$, sin tratamiento previo, $\mathrm{n}(\%)$ & $4(7,8)$ & N/A & N/A & $\mathrm{N} / \mathrm{A}$ & $\mathrm{N} / \mathrm{A}$ \\
\hline No OP-P, n (\%) & $3(5,9)$ & $\mathrm{N} / \mathrm{A}$ & $5(9,80)$ & $\mathrm{N} / \mathrm{A}$ & $1(1,9)$ \\
\hline No OP-P, sexo masculino, n (\%) & $0(0,0)$ & N/A & $4(7,8)$ & N/A & $11(21,2)$ \\
\hline No OP-P, sexo masculino, sin fractura previa, $\mathrm{n}(\%)$ & $1(2,0)$ & N/A & $2(3,9)$ & N/A & $0(0,0)$ \\
\hline No OP-P, sexo masculino, sin tratamiento previo, $\mathrm{n}(\%)$ & $2(3,9)$ & N/A & $\mathrm{N} / \mathrm{A}$ & $\mathrm{N} / \mathrm{A}$ & $9(17,3)$ \\
\hline No OP-P, sexo masculino, sin fractura previa, sin tratamiento previo, $\mathrm{n}(\%)$ & $1(2,0)$ & $\mathrm{N} / \mathrm{A}$ & $\mathrm{N} / \mathrm{A}$ & $\mathrm{N} / \mathrm{A}$ & $\mathrm{N} / \mathrm{A}$ \\
\hline No OP-P, sin fractura previa, n (\%) & $0(0,0)$ & $\mathrm{N} / \mathrm{A}$ & $2(3,9)$ & $\mathrm{N} / \mathrm{A}$ & $0(0,0)$ \\
\hline No OP-P, sin tratamiento previo, n (\%) & $1(2,0)$ & N/A & N/A & N/A & $2(3,9)$ \\
\hline Sin fractura previa, $\mathrm{n}(\%)$ & $1(2,0)$ & N/A & $6(11,8)$ & N/A & N/A \\
\hline Sin tratamiento previo, $\mathrm{n}(\%)$ & $12(23,5)$ & N/A & N/A & N/A & $4(7,7)$ \\
\hline Sin fractura previa, sin tratamiento previo, $\mathrm{n}(\%)$ & $2(3,9)$ & N/A & N/A & N/A & N/A \\
\hline Ausencia global de concordancia por guía, n (\%) & $38(74,5)$ & $2(3,9)$ & $19(37,2)$ & $3(5,8)$ & $27(51,9)$ \\
\hline Ausencia de concordancia a las TRES guías por medicamento, n (\%, valor de $\mathrm{p})$ & \multicolumn{3}{|c|}{$38(74,5)$} & \multicolumn{2}{|c|}{$27(51,9)$} \\
\hline - Ausencia de concordancia a las TRES guías por medicamento, femenino, n (\%) & \multicolumn{2}{|c|}{$32(62,7)$} & $\mathrm{p}=0,375$ & \multicolumn{2}{|c|}{$7(13,5)$} \\
\hline - Ausencia de concordancia a las TRES guías por medicamento, masculino, n (\%) & \multicolumn{2}{|c|}{$6(11,8)$} & $\mathrm{p}=0,686$ & \multicolumn{2}{|c|}{$20(38,5)$} \\
\hline Ausencia de concordancia a las TRES guías de los dos medicamentos, n (\%) & & & $65(66,1)$ & & \\
\hline
\end{tabular}

${ }^{a}$ National Osteoporosis Foundation

${ }^{b}$ National Institute for Health and Clinical Excellence

${ }^{\mathrm{c} S}$ Scottish Intercollegiate Guidelines Network

*La Guía NICE no especifica información relacionada con el ácido zoledrónico.

Siglas: N/A: No aplica, OP-P: Osteoporosis posmenopáusica, DMO: Densitometría mineral ósea. 


\section{Discusión}

Este estudio evaluó la concordancia en la utilización de los medicamentos teriparatide y ácido zoledrónico con las recomendaciones de tres guías internacionales $\left(\mathrm{NOF}^{19}, \mathrm{NICE}^{20}\right.$ y $\mathrm{SIGN}^{21}$ ), y mostró que sólo el 36,9\% de los casos se ajustaron a dichas recomendaciones. Es importante señalar, que las guías de la NICE y SIGN limitan el uso de ambos medicamentos a pacientes con diagnóstico de osteoporosis posmenopáusica; lo que podría explicar que, las principales causas de falta de concordancia o prescripción inadecuada se asocien al uso en diagnósticos diferentes a osteoporosis posmenopáusica y al sexo masculino. Sin embargo, las indicaciones autorizadas en Colombia, según el registro sanitario expedido por el Instituto Nacional de Vigilancia de Medicamentos y Alimentos (INVIMA), son más amplias para $\mathrm{TTP}^{22}$ y para $\mathrm{Zol}^{23}$, y se ajustan más a las recomendaciones de la guía $\mathrm{NOF}^{19}$; por ello, los porcentajes de concordancia, acorde con esta guía, fueron mayores: TTP: 96,1\% y Zol: 94,2\%.

El uso de TTP o Zol como terapia inicial fue otra de las causas importantes de ausencia de concordancia. En este contexto, es importante aclarar que, en caso de contraindicación o de un riesgo elevado de fracturas a 10 años (ej. Fractura de cadera $\geq 3 \%$ o fractura osteoporótica mayor $\geq 20 \%$ ), se justificaría el uso de estos fármacos como primera opción. Sin embargo, en la historia clínica no se identificó alguna contraindicación para el tratamiento inicial con bifosfonatos, recomendaciones de las guías $\mathrm{NICE}^{20}$ y $\mathrm{SIGN}^{21}$. No obstante, este hallazgo podría deberse a subregistro de la información o que se encuentre en una fuente a la que no se haya tenido acceso. En este sentido, es importante aclarar que, algunos pacientes estaban previamente afiliados en otras instituciones/aseguradoras o fueron atendidos por especialistas de otras instituciones de salud diferentes a las revisadas.

Otra causa de no concordancia fue el uso de TTP o Zol en prevención primaria (uso en pacientes sin fractura previa $)^{21}$. De forma específica, al $57,3 \%$ de los pacientes se les prescribió estos medicamentos para prevención primaria. En el caso del Zol, el 75,0\% de los casos se encontró ajustado a las recomendaciones de las guías $\mathrm{NOF}^{19}$ y SIGN ${ }^{21}$ para esta indicación (la NICE no incluye el Zol). Por su parte, para la TTP, en el 19,6\% de los casos no fue adecuado el uso, basado en las recomendaciones de la $\mathrm{NICE}^{20}$ y la SIGN $^{21}$.

La población de estudio corresponde en su mayoría al sexo femenino y principalmente con diagnóstico de osteoporosis posmenopáusica, grupo poblacional en el que es más prevalente esta enfermedad ${ }^{1,19}$, la incidencia de fracturas es dos a tres veces mayor que en los hombres ${ }^{24}$, existe mayor evidencia en términos de eficacia y seguridad que soportan las opciones terapéuticas disponibles ${ }^{25}$; por tanto, se encontró un consenso en las tres guías revisadas, que avalan el uso de TTP y Zol en dicha población. Al estratificar las variables asociados a factores de riesgo de la OMS y ausencia de concordancia por sexo, sólo se identificó diferencias estadísticamente significativas en la variable "fracturas previas", la cual se presentó en mayor proporción para el sexo femenino que fue tratado con TTP; sin embargo, al realizar el cálculo de riesgo de fractura mayor o de cadera utilizando el algoritmo FRAX de la $\mathrm{OMS}^{17}$, el cual incluye para su cálculo dicha variable, no se encontraron diferencia por grupo de medicamento ni por sexo, Tabla 3.

En este estudio, se identificaron tres pacientes $(5,9 \%)$ que superaron la duración máxima de dos años de tratamiento, durante toda la vida, recomendado con TTP, debido a un mayor riesgo de osteosarcoma ${ }^{20}$. De forma similar, en el caso del Zol, se identificaron cuatro pacientes $(7,7 \%)$ que superaron los tres años máximo de tratamiento recomendado, debido a que el aumento de eficacia es dudoso, pero el riesgo de reacciones adversas es mayor ${ }^{26}$. Este grupo de pacientes, debido a la intervención del farmacéutico, se les realizó cambio y/o suspendió el tratamiento.

En un estudio realizado por Moncayo, et al. ${ }^{15}$, en el que se evaluó el uso de diferentes medicamentos utilizados para el tratamiento de la osteoporosis y osteopenia en una institución de Bogotá-Colombia, el uso de TTP fue acorde con las recomendaciones de las guías NOF y NICE en $100 \%$ y $40 \%$, respectivamente. Por su parte, en el presente estudio la concordancia fue del $96,1 \%$ y $25,5 \%$, respectivamente. También, los autores reportaron que el $85,7 \%$ de los pacientes con TTP fueron tratados previamente con otro medicamento, comparado con el 54,9\% hallado en este estudio. Sin embargo, en el estudio de Moncayo, et al. ${ }^{15}$ no se especificó el número de pacientes que utilizaron este medicamento, lo que dificulta identificar posibles explicaciones a las diferencias encontradas en los dos estudios. Adicionalmente, en el estudio de Moncayo, et al. ${ }^{15}$ no se especifican los datos para el Zol, son presentados para los bifosfonatos como grupo.

Por su parte, un estudio realizado en España por Idarreta, et al. ${ }^{27}$, señala que el $30,6 \%$ de las prescripciones de fármacos para la osteoporosis no tienen indicación basada en la evidencia. Sin embargo, estos resultados no son comparables con los hallazgos del presente 
estudio, debido a el tamaño de la muestra (512 vs 103), los medicamentos revisados (no se incluye el Zol, y sólo cinco pacientes estaban en tratamiento con TTP), y la diferencia en los parámetros valorados para establecer prescripción basada en evidencia. No obstante, los autores señalan un elemento a superar en el caso de los pacientes con osteoporosis, como lo es la ausencia de consenso entre las sociedades científicas y las guías de práctica clínica, debido a que el diagnóstico y la indicación de tratamiento para la osteoporosis son confusos, algunas veces puede estar basado en la presencia de ciertos factores de riesgo, resultados de la densitometría u otras razones ${ }^{27}$.

El presente estudio presenta algunas limitaciones, por lo que los resultados y conclusiones deben utilizarse con precaución. El más notorio corresponde al tipo de estudio (descriptivo-retrospectivo), basado en la revisión de la historia clínica como fuente principal de los datos recolectados, lo que genera algunas dudas importantes con la información no identificada. En este sentido, se destacan: 1) En el 29,1\% de los casos no se accedió a los resultados de DMO de cuello femoral, y se desconoce si corresponde a un subregistro o si se inició tratamiento con alguno de los dos fármacos evaluados, sólo con el soporte de los factores de riesgo. 2) Para ningún paciente se registró la presencia de antecedentes de fracturas de los padres, parámetro evaluado para el cálculo de riesgo de fractura a 10 años- FRAX® 3) Para todos los pacientes no se logró identificar las causas de cambio o suspensión del tratamiento. Otra limitación del presente estudio es que los datos de los pacientes, aunque provienen de 22 de las principales regiones de Colombia, son de una sola empresa de salud (asegurador), lo que dificulta la extrapolación de los resultados encontrados a la población colombiana en general. Sin embargo, a pesar de estas limitaciones, se destaca la importancia de los estudios de utilización de medicamentos, ya que pueden aportar a su utilización adecuada. En el caso de la osteoporosis, las guías de manejo contemplan el análisis de múltiples factores para su diagnóstico y tratamiento, lo cual puede generar confusión a la hora de seleccionar el tratamiento más apropiado y según las características particulares de cada paciente ${ }^{15,27}$. Por ello, es necesario definir unos lineamientos claros e uniformes entre las instituciones y grupos de profesionales responsables de la atención en salud, basados en guías internacionales y la mejor evidencia clínica ${ }^{28}$ y a así, evitar malgastar recursos en salud que cada vez son más limitados, ya sea por uso de un medicamento no indicado, que para el caso de TTP y Zol son considerados como de alto costo, o por los riesgos asociados a problemas de seguridad.

\section{Conclusiones}

En este grupo limitado de pacientes, sólo en el 36,9\% de los casos revisados, la utilización de teriparatide o ácido zoledrónico se ajusta a las guías de organizaciones internacionales. La ausencia de concordancia se debe especialmente a:

- Uso en diagnósticos diferentes a osteoporosis posmenopáusica $(43,7 \%)$.

- Uso como terapia inicial $(36,9 \%)$.

- Sexo masculino (30,1\%).

- Uso en prevención primaria, principalmente teriparatide $(21,4 \%)$.

\section{Agradecimientos}

A Audifarma S.A. por el apoyo en la realización de este trabajo.

\section{Consideraciones éticas}

Este estudio se clasificó en la categoría de "investigación sin riesgo", según la resolución No. 8430 de 1993 del Ministerio de Salud de Colombia, que establece las normas científicas, técnicas y administrativas para la investigación en salud y se ajustó a los principios de beneficencia, y confidencialidad de la Declaración de Helsinki. Además el estudio fue avalado por el Comité de Investigaciones de Audifarma S.A. y autorizado por la institución de salud responsable de la atención de los pacientes.

\section{Conflicto de interés}

El medicamento teriparatide utilizado en este grupo de pacientes corresponde a la molécula innovadora (Forteo ${ }^{\circledR)}$ del laboratorio Eli Lilly, sin embargo, se aclara que no se tiene ningún vínculo ni se contó con la financiación de dicho laboratorio para la elaboración de este trabajo. Por su parte, para el principio activo ácido zoledrónico, se utilizó tanto el medicamento innovador como genérico. Los autores Yuly Henao, Ilsa Parrado y Piedad Botero trabajan para Audifarma, gestor de servicios farmacéuticos cuya relación con los laboratorios fabricantes de los medicamentos estudiados es de cliente-proveedor. Pedro Amariles no tiene ningún conflicto de interés.

\section{Referencias}

1. Migliore A, Broccoli S, Massafra U, Cassol M, Frediani B. Ranking antireabsorptive agents to prevent 
vertebral fractures in postmenopausal osteoporosis by mixed treatment comparison meta-analysis. Eur Rev Med Pharmacol Sci. 2013; 17(5): 658-667.

2. World Health Organization. World report on aging and health. WHO Library cataloguing-inpublication Data. 2015, WT104; 53-54.

3. Zanchetta J, MacDonald S. The Latin America Regional Audit Epidemiología, costos e impacto de la osteoporosis en 2012. International Osteoporosis Foundation; 2012.

4. Carmona F. Osteoporosis en Santafé de Bogotá, Instituto Nacional de Salud de Colombia; 1999.

5. Morales-Torres J, Gutiérrez-Ureña S. Osteoporosis Committee of Pan-American League of Associations for Rheumatology. The Burden of Osteoporosis in Latin America. Osteoporos Int. 2004; 15: 625-632. DOI: 10.1007/s00198-004-1596-3.

6. Black DM, Cummings SR, Karpf DB, Cauley JA, Thompson DE, Nevitt MC, et al. Randomised trial of effect of alendronate on risk of fracture in women with existing vertebral fractures. Fracture Intervention Trial Research Group. Lancet. 1996; 348(9041): 1535-1541. DOI: http://dx.doi. org/10.1016/S0140-6736(96)07088-2

7. Harris ST, Watts NB, Genant HK, Mckeever CD, Hangartner T, Keller M, et al. Effects of risedronate treatment on vertebral and nonvertebral fractures in women with postmenopausal osteoporosis: a randomized controlled trial. Vertebral Efficacy With Risedronate Therapy (VERT) Study Group. JAMA. 1999; 282(14): 1344-1352. DOI: 10.1001/ jama.282.14.1344.

8. McClung MR, Wasnich RD, Recker R, Cauley JA, Chesnut CH 3rd, Ensrud Ke, et al. Oral Ibandronate Study Group. Oral daily ibandronate prevents bone loss in ear ly postmenopausal women without osteoporosis. J Bone Miner Res. 2004; 19(1): 1118. DOI:10.1359/JBMR.0301202.

9. Black DM, Delmas PD, Eastell R, Reid IR, Boonen S, Cauley JA, et al. Horizon Pivotal Fracture Trial. Once-yearly zoledronic acid for treatment of postmenopausal osteoporosis. N Engl J Med. 2007; 356(18): 1809-1822. DOI: 10.1056/ NEJMoa067312.

10. Lyles KW, Colón-Emeric CS, Magaziner JS, Adachi JD, Pieper CF, Mautalen C et al. HORIZON Recurrent Fracture Trial. Zoledronic acid and clinical fractures and mortality after hip fracture. N Engl J Med. 2007; 357(18): 1799-1809. DOI: 10.1056/NEJMoa074941.

11. Zhang L, Pang Y, Shi Y, Xu M, Xu X, Zhang J, et al. Indirect comparison of teriparatide, denosumab, and oral bisphosphonates for the prevention of vertebral and nonvertebral fractures in postmenopausal women with osteoporosis. Menopause. 2015; 22(9): 10211025. DOI: $10.1097 / G M E .0000000000000466$.

12. Liu H, Michaud K, Nayak S, Karpf DB, Owens DK, Garber AM. The cost-effectiveness of therapy with teriparitide and alendronate in women with severe osteoporosis. Arch Intern Med. 2006; 166(11): 1209-1217. DOI: 10.1001/archinte.166.11.1209.

13. Panico A, Lupoli GA, Marciello F, Lupoli R, Cacciapuoti M, Martinelli A, et al. Teriparatide vs. Alendronate as a treatment for osteoporosis: Changes in biochemical markers of bone turnover, BMD and quality of life. Med Sci Monit. 2011; 17(8): CR442-448.

14. Hodsman AB, Bauer DC, Dempster DW, Dian L, Hanley DA, Harris ST et al. Parathyroid hormone and teriparatide for the treatment of osteoporosis: a review of the evidence and suggested guidelines for its use. Endocrine Rev. 2005; 26(5): 688-703. DOI: https://doi.org/10.1210/er.2004-0006.

15. Moncayo S, López J. Evaluación del manejo farmacológico de la osteoporosis y la osteopenia en una institución de régimen especial de Bogotá. Rev. salud pública. 2015; 17(4): 565-577. DOI: https:// doi.org/10.15446/rsap.v17n4.38450.

16. Mayor S, Velandia D. Análisis de impacto presupuestal de teriparatide para reducir la frecuencia de fracturas vertebrales y no vertebrales, en adultos con riesgo elevado de fractura. Bogotá-Colombia: Instituto de Evaluación Tecnológica en Salud; 2014.

17. FRAX ${ }^{\circledR}$ : The World Health Organization Fracture Risk Assessment Tool.

18. Looker AC1, Orwoll ES, Johnston CC Jr, Lindsay RL, Wahner HW, Dunn WL, et al. Prevalence of low femoral bone density in older U.S. adults from NHANES III. J Bone Miner Res. 1997; 12(11): 1761-1768. DOI: 10.1359/jbmr.1997.12.11.1761.

19. National Osteoporosis Foundation. Clinician's Guide to Prevention and Treatment of Osteoporosis. Washington: National Osteoporosis Foundation; 2010.

20. National Institute for Health and Clinical Excellence. Osteoporosis: assessing the risk of fragility fracture. Manchester: NICE guideline; 2012.

21. Scottish Intercollegiate Guidelines Network (SIGN). Management of osteoporosis and the prevention of fragility fractures. Edinburgh: SIGN; 2015.

22. Instituto Nacional de Vigilancia de Medicamentos y Alimentos (INVIMA). Registro sanitario: Teriparatide. Colombia; 2015.

23. Instituto Nacional de Vigilancia de Medicamentos y Alimentos (INVIMA). Registro sanitario: Ácido Zoledrónico $5 \mathrm{mg}$.

24. González L, Vásquez G, Molina J. Epidemiología 
de la osteoporosis. Rev Colomb Reumatol. 2009; 6(1): 61-75.

25. Kaufman JM, Lapauw B, Goemaere S. Current and future treatments of osteoporosis in men. Best Pract Res Clin Endocrinol Metab. 2014; 28(6): 871-884. DOI: 10.1016/j.beem.2014.09.002.

26. Black DM, Reid IR, Boonen S, Bucci-Rechtweg C, Cauley JA, Cosman F, et al. The effect of 3 versus 6 years of zoledronic acid treatment of osteoporosis: a randomized extension to the HORIZON-Pivotal Fracture Trial (PFT). J Bone Miner Res. 2012; 27(2): 243-254. DOI: 10.1002/jbmr. 1494.

27. Idarreta I, Rua E, Hernando F, Zabaleta K, Arzuaga M, Irizar M. Consumidores, prescripción y prescriptores de fármacos para la osteoporosis. ACMB. 2010; 107(3): 90-96. DOI: 10.1016/S0304-4858(10)70024-X

28. Machado J, Moreno P, Bañol A. Patrones de prescripción de broncodilatadores y corticoides inhalados en pacientes adultos de Colombia. Acta Med Colomb. 2015; 40(3): 218-226. 